\title{
Analysis of Influence of Scale Factor and Design on Performance Characteristics and Stress Strain State of Micro Sensing Element
}

\author{
Mikhail Kotelnikov ${ }^{1}$, Aleksey Schenyatskiy ${ }^{1}$, and Anna Basharova ${ }^{1, *}$ \\ ${ }^{1}$ Kalashnikov Izhevsk State Technical University, Izhevsk, Russia
}

\begin{abstract}
A new type of semitoroidal type sensing element is discussed in this scientific article. In the study, possible modes of vibration were identified and a working one was selected. A dynamic analysis was carried out and it was established what force is necessary to excite the resonator in order to ensure both the vibration amplitude that is convenient for reading, and the strength of the resonator. The study of the influence of the scale factor on the performance characteristics and stress strain state of the micro SE was carried out. The SE sizes were changed proportionally. The simulation was carried out with seven options concerning sizes, the calculation was performed with the help of the finite element method, for each individual case, and the results were tabulated. Based on the results obtained, graphs were plotted and conclusions were drawn concerning the influence of the scale factor on the performance characteristics and stress strain state of the device. After that, tasks were identified for further study of micro SE, as an advanced model. Finally, the references including eight sources were given.
\end{abstract}

High standards for sensing elements (SE) of control systems related to mobile units (MU) have led to the creation of unconventional gyroscopic devices based on new physical principles developed from the creation of natural frequencies and deflection measurements. Such devices include a solid-state wave gyroscope (SSWG) with a quartz crystal semitoroidal resonator, the operation of which is based on the use of the inert properties of standing waves excited by the vibrating edges of an axisymmetric resonator [1-4]. Therefore, it is relevant to study the influence of materials and geometric parameters of a SE made in the form of a semitoroid, on its technical parameters.

In this scientific article, the influence of the scale factor and design on the performance characteristics and stress strain state of a micro SE on its natural frequencies is analyzed. The research was carried out as follows.

At the first stage, the natural frequencies of the resonator and possible modes of vibration were calculated.

During the operation of the resonator, forces harmonically varying according to a certain law are applied to it, thereby forcing it to deform and vibrate. The values of deformations and their distribution over the body of the SE are determined by its geometric

*Corresponding author: phoenix-anya@mail.ru 
parameters and materials used $[5,6]$. In addition, at different frequencies the directions and values will differ significantly. Consequently, it is necessary to conduct a modal analysis of the design of a semitoroidal resonator, as a result of which it is necessary to obtain the natural frequencies values at various modes of vibration and to choose the most suitable one for control and information retrieval.

The study was carried out by the finite element method in the CAE ANSYS program $[7,8]$. In order to perform this, a resonator model (Figure 1a) with the main geometric parameters listed in Table 2, Figure 1b, was taken. The quartz glass with the characteristics listed in Table 1 was chosen as the material of the resonator.

Table 1. Quartz Glass Characteristics.

\begin{tabular}{|c|c|c|}
\hline No. & Name & Amount \\
\hline 1 & Density & $2.2 \mathrm{gr} / \mathrm{cm}^{3}$ \\
\hline 2 & Young's Modulus & $7.5 \times 10^{10} \mathrm{~N} / \mathrm{m}^{2}$ \\
\hline 3 & Poisson's Ratio & 0.19 \\
\hline 4 & Ultimate Resistance & $39 \mathrm{MPa}$ \\
\hline 5 & Coefficient of Internal Friction & $10^{-4}$ \\
\hline
\end{tabular}

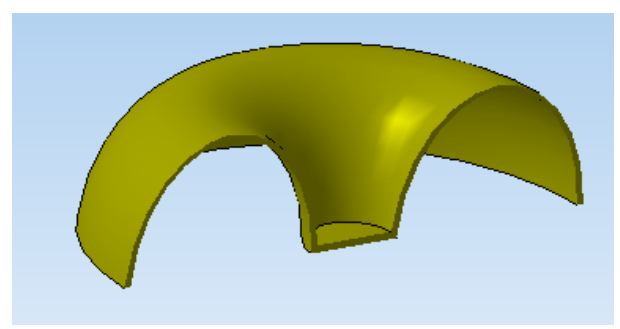

a

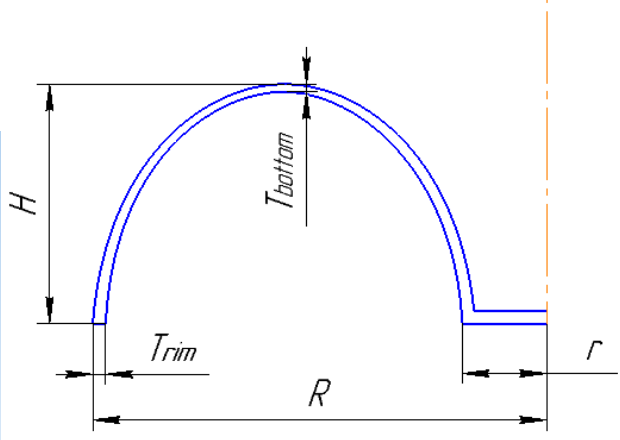

b

Fig. 1. Semitoroidal resonator. $\mathrm{a}-3 \mathrm{D}$ cut-away model, $\mathrm{b}-$ main geometric parameters.

Table 2. Geometric parameters of resonator model.

\begin{tabular}{|c|c|}
\hline Parameter & Amount \\
\hline $\mathrm{R}$ & $3.5 \mathrm{~mm}$ \\
\hline $\mathrm{r}$ & $0.55 \mathrm{~mm}$ \\
\hline $\mathrm{H}$ & $1.85 \mathrm{~mm}$ \\
\hline $\mathrm{T}_{\text {rim }}$ & $100 \mu \mathrm{m}$ \\
\hline $\mathrm{T}_{\text {bottom }}$ & $61 \mu \mathrm{m}$ \\
\hline
\end{tabular}

The resonator in the sensor will be fixed to the base of the inner surface, and the moving element will be represented by the outer surface. The resonator fixation ensures the immobility of the surface in the clamping area in the radial, circumferential and axial directions. Based on the above, the analysis is performed. To obtain more reliable information when modeling and performing calculations, finite elements fine grid is performed in the zones of geometry change. The smallest finite element size makes 0.025 $\mathrm{mm}$. Figures $2 \mathrm{a}-2 \mathrm{~d}$ show possible modes of vibration. 


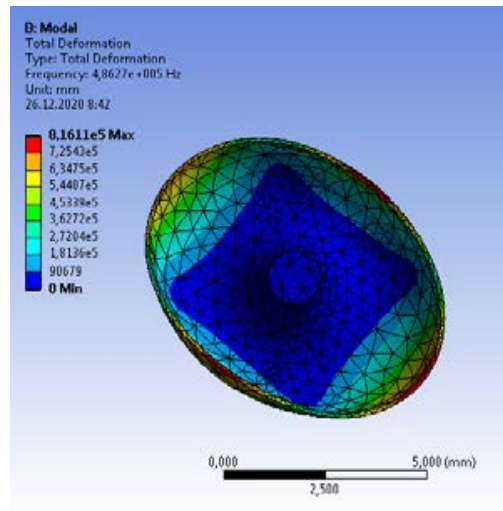

$\mathrm{a}$

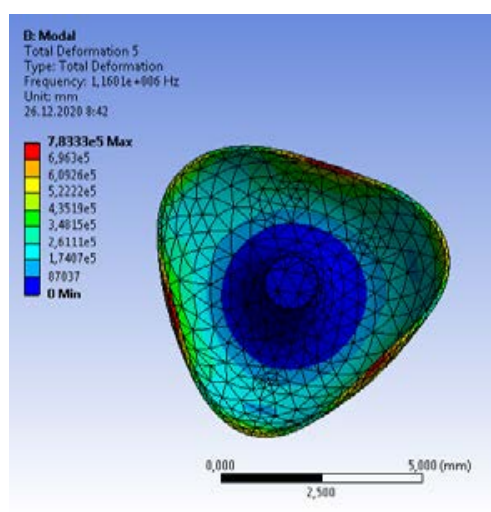

$\mathrm{c}$

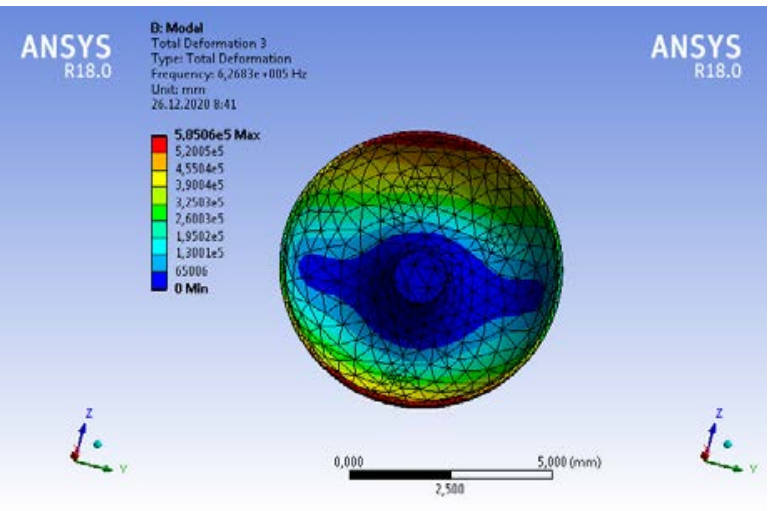

b

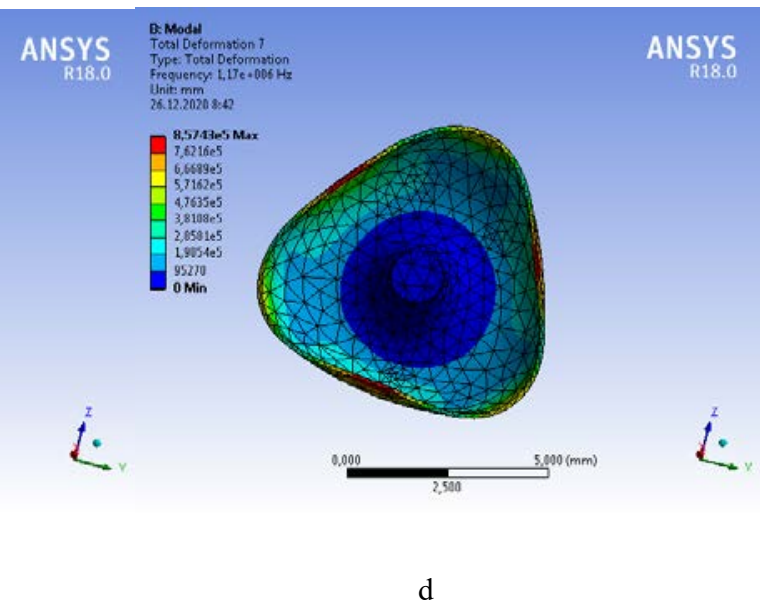

Fig. 2. Possible modes of vibration: a - mode of vibration $1, \mathrm{~b}$ - mode of vibration $3, \mathrm{c}-$ mode of vibration $5, \mathrm{~d}-$ mode of vibration 7 .

Due to the fact that mode of vibration 1 is the most suitable one for controlling and reading information, further research will be carried out with this mode at its natural frequency of $11.8 \mathrm{kHz}$.

At the second stage, dynamic analysis was carried out.

Statement of the problem:

1. The resonator is fixed by its inner part.

2. Excitation is carried out in accordance with the first mode with its natural frequency that makes $11.8 \mathrm{kHz}$.

3. The resonator is made of a homogeneous material with no technological defects.

4. Excitation forces are applied to the edges of the outer shell of the resonator.

5. It is necessary to determine maximum stresses and displacements of the moving element, with the applied force that makes $1.5 \times 10^{-6} \mathrm{~N}$.

Results of the analysis:

Figure 3 shows the numerical values of the maximum stresses and their concentration, and the maximum displacements of the moving elements are shown. Figure 4 shows the maximum movements of the moving elements. 


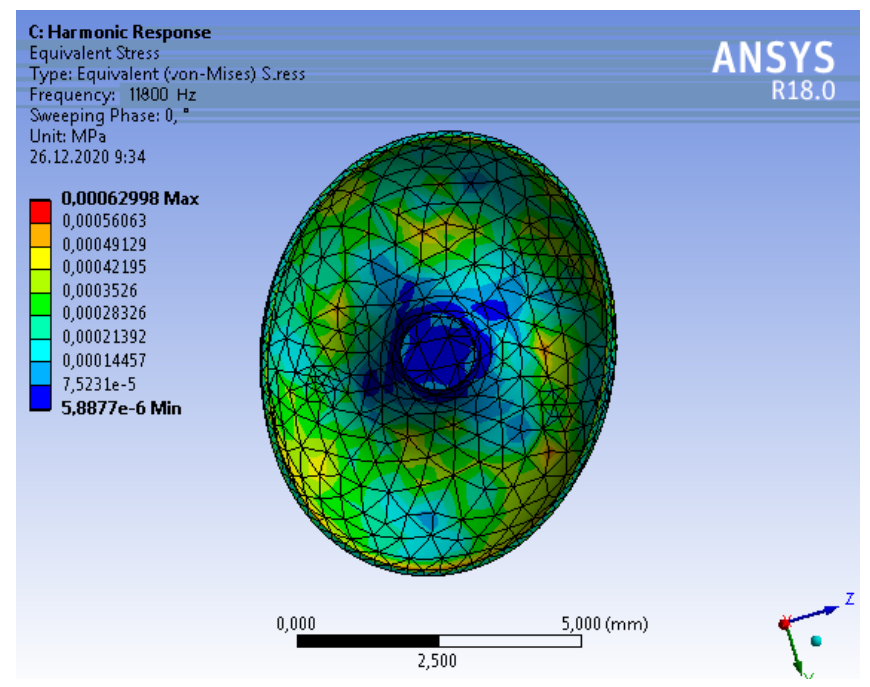

Fig. 3. Maximum stresses.

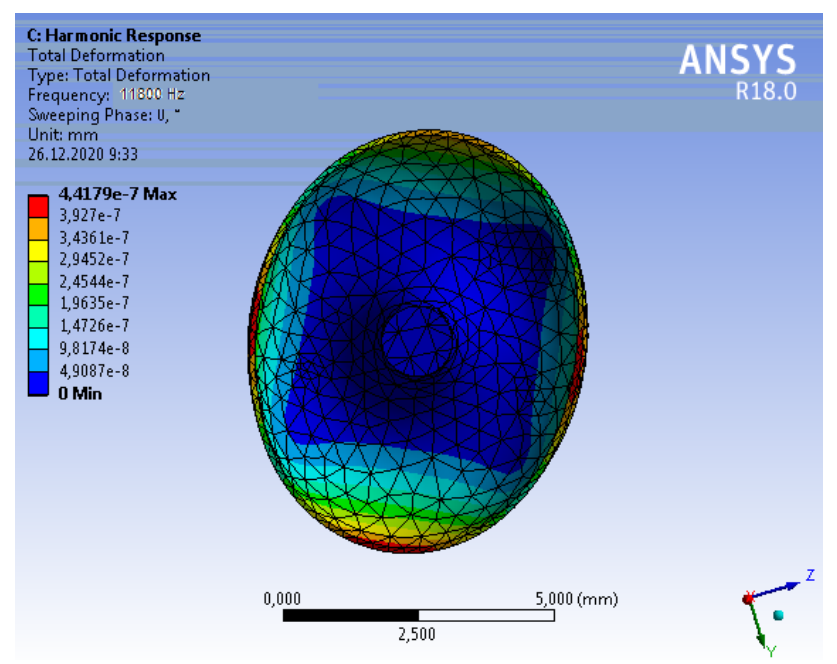

Fig. 4. Maximum displacements.

Based on the combined results obtained, it is possible to state that excitation with the applied force that makes $1.5 \times 10^{-6} \mathrm{~N}$, will be optimal and safe, since it is assumed that a relatively small stress is required to obtain this force, and the part will have a low stress, thereby guaranteeing the integrity of the resonator during operation. Moving elements will move with the amplitude of $0.44 \mu \mathrm{m}$.

The analysis carried out in the work shows that, on the one hand, it is possible to make a resonator for carrying out an experiment, since a small force is required to excite it. Moreover, the resonator is also able to withstand a load at this force. On the other hand, further study is required to make such a simulation with a through hole in the base, fixing both the inner part and the outer part and at the same time changing the scale factor.

At the third stage, modal analysis was carried out by changing the design of the resonator by adding a hole in the base. The scale factor was changed with epy step of $0.25 \mathrm{~d}$ 
$(\mathrm{d}=2 \mathrm{R})$. The results of the analysis were listed in Table 3. The modeling was carried out with the fixation of the resonator both to the inner part and to the outer one. Figure 5 shows the calculated natural frequency at $\mathrm{d}=1$.

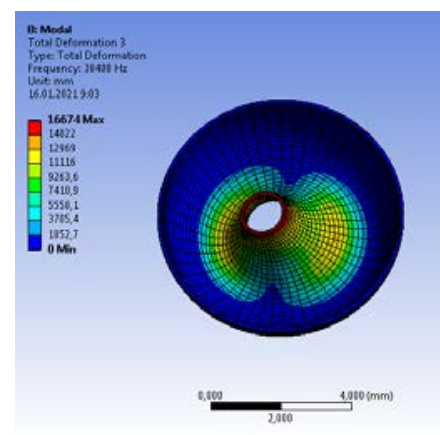

a
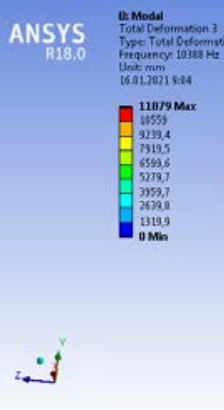

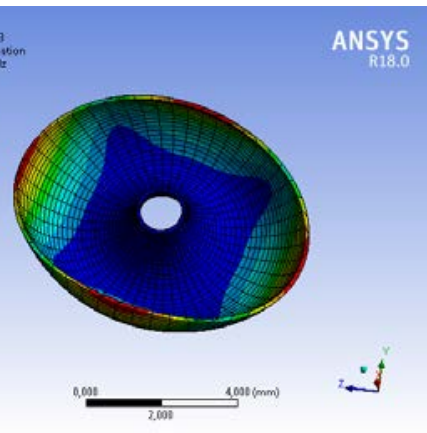

$\mathrm{b}$

Fig. 5. Calculated natural frequencies. $a-$ when fixed to the outer part, $b$ - when fixed to the inner part.

Table 3. Modal Analysis Results.

\begin{tabular}{|c|c|c|}
\hline Size of a SE & $\begin{array}{c}\text { Vibration frequency with internal } \\
\text { fixation, } \mathbf{v}(\mathbf{k H z})\end{array}$ & $\begin{array}{c}\text { Vibration frequency with external } \\
\text { fixation, } \mathbf{v}(\mathbf{k H z})\end{array}$ \\
\hline $0.5 \mathrm{~d}$ & 20.8 & 76.8 \\
\hline $0.75 \mathrm{~d}$ & 13.9 & 51.2 \\
\hline $\mathrm{d}$ & 11.8 & 30.7 \\
\hline $1.25 \mathrm{~d}$ & 8.3 & 16.7 \\
\hline $1.5 \mathrm{~d}$ & 6.9 & 9.1 \\
\hline $1.75 \mathrm{~d}$ & 5.1 & 7.2 \\
\hline $2 \mathrm{~d}$ & 4.2 & 5.9 \\
\hline
\end{tabular}

Based on Table 3, the graphs of the dependence of the resonator natural frequency on the dimensions, when attached to the outer part (Figure 6) and the dependence of the resonator natural frequency on the dimensions, when attached to the inner part (Figure 7), are plotted.

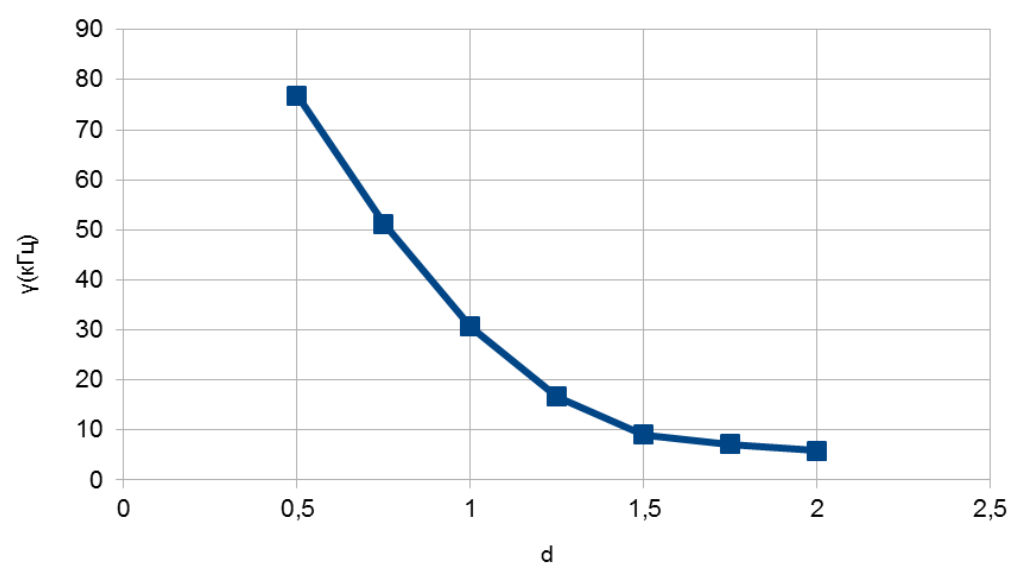

Fig. 6. Dependence of the resonator natural frequency on the dimensions, when attached to the outer part. 


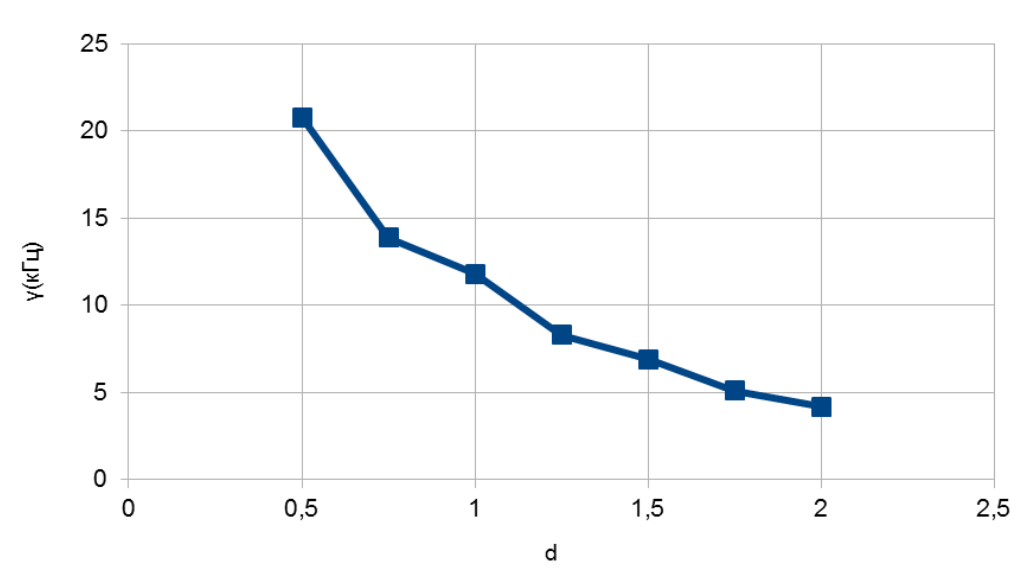

Fig. 7. Dependence of the resonator natural frequency on the dimensions, when attached to the inner part.

The estimation of the resonator natural frequencies with a proportional change in the dimensions shows that with an increase in the dimensions of the resonator, the natural frequencies decrease. With internal fixation, the dependence is linear, and with external fixation, the dependence is exponential.

\section{Conclusion}

In the article, the natural frequencies, the total amplitude of vibrations of the sphere, and also the maximum stresses were determined on the basis of the carried out modal and dynamic analysis of a semitoroidal resonator. With the sequential performance of three stages of the study, possible modes of vibration were established and the working one with the natural frequency of $11.8 \mathrm{kHz}$ was chosen. Then a dynamic analysis was carried out, in which it was revealed that, to excite the resonator, it was necessary and sufficient to apply the force of $1.5 \times 10^{-6} \mathrm{~N}$. Thus, both the amplitude of vibration, which is convenient for reading, and the strength of the resonator will be ensured. From here on, the laws of change in natural frequencies from changes in the size of the resonator were determined. It was found that with an increase in the size of the resonator, the natural frequencies decreased; when fixed to the inner part, the dependence was linear, and when fixed to the outer part of a semitoroidal resonator, the dependence was exponential.

\section{References}

1. N. Mehanathan, V. Tavassoli, P. Shao, L. Sorenson and F. Ayazi, Invar-36 Micro Hemispherical Shell Resonators, in IEEE 27th Int. Conf. on Micro Electro Mechanical Systems, San-Francisco, California, the USA, January 2014, P. 40-43.

2. L.D. Sorenson, X. Gao, F. Ayazi. 3-D Micromachined Hemispherical Shell Resonators with Integrated Capacitive Transducers, 2012 IEEE 25th International Conference on Micro Electro Mechanical Systems (MEMS), Paris, France, 2012, P. 168-171.

3. D. Xiao et al. Thin-Walled Resonator Made of Fused Silica with T-Shaped Masses Used in a Gyroscope, J. Microelectromech. Syst. Volume 27, No. 1, pp. 47-58, February 2018. 
4. Bryan, G.H. On the Beats in the Vibrations of a Revolving Cylinder or Bell // Proc. Camb. Phil. Soc. - 1890. - Vol. 7. - P. 101-111.

5. M.A. Kotelnikov, A.A. Basharova, P.V. Lekomtsev, A.V. Schenyatskiy. Statement of Mathematical Modeling Problem of a Rod Sensing Element of a Solid-State Wave Gyroscope // Intelligent Systems in Production. - 2016. - P. 69-73.

6. M.A. Kotelnikov, A.B. Schenyatskiy, A.A. Basharova. Influence of Sensing Element Design Parameters on Technical Characteristics of a Solid-State Wave Gyroscope // Bulletin of Perm National Research Polytechnic University. Mechanical Engineering, Materials Science. - 2017. - Volume 19, No. 2. - P. 92-106.

7. Zenkevich, O. Finite Element Method in Engineering / Transl. from English. - Edited by B.E. Pobedri. - M.: Mir, 1975. - 541 p.

8. Kaplun, A.B., Morozov, Ye.M., Olferyeva, M.A. ANSYS in the Hands of an Engineer: A Practical Guide. - M.: Editorial URSS, 2003. - 272 p. 